\title{
Vehicle Target Detection Based on Improved Mixed Gaussian Modeling
}

\author{
Caiyun Xie ${ }^{1}$, Ruxia Hong ${ }^{1, *}$, Jing Wang ${ }^{2}$ and Hong $\mathrm{Yu}^{1}$ \\ ${ }^{1}$ YuZhang Normal University, Nanchang, Jiangxi, China \\ ${ }^{2}$ Information Electromechanical Department, The Board of the Middle Route Project of South-to-North Water Diversion Project, \\ Zhengzhou, Henan, China \\ ${ }^{*}$ Corresponding author
}

\begin{abstract}
In the modern world, with the rapid development of science and technology, computer and related technologies have been used in many fields; of course, intelligent video surveillance system cannot do without computer technology. This system also cannot do without the core issue: the detection of the goal vehicle. Only the smooth detection of moving vehicles can complete the intelligent video surveillance system speed. This paper mainly introduces several methods of vehicle target detection, and provides the advantages and disadvantages of every method, and improves the traditional mixed Gaussian modeling method. The steps required to complete this method are introduced in detail.
\end{abstract}

Keywords-gaussian modeling; video speed; vehicle target detection component

\section{INTRODUCTION}

With the rapid economic growth, the number of vehicles in our country has risen sharply, which has also helped to gradually expand the scale of our country's highways. Therefore, the annual number of traffic accidents in our country also increases. In order to effectively reduce traffic accidents and improve traffic quality, it is necessary to introduce intelligent monitoring system to monitor the traffic and traffic conditions in real time through the intelligent traffic system so as to solve the problem of traffic disturbance in time and improve the processing efficiency of related parts and avoid unnecessary troubles. In the field of intelligent transportation, the term "video tachymeter" has also appeared frequently in recent years as a hot spot. Video tachometer is a very costeffective technology that can keep abreast of traffic conditions only through the camera without installing radar and sensors. Therefore it is also widely used.

In the intelligent monitoring system, the most critical and foremost step is to detect the target of a moving vehicle. By detecting a series of images, dynamic pixels and static pixels are respectively obtained, and then the changed regions are extracted from the pixels. By judging the detected moving pixels to draw whether we need to deal with the unexpected situation. The accuracy vehicle target detection of the latter part of the video speed is crucial; it is the first step in the completion of video speed measurement.

\section{INTRODUCTION OF VEHICLE TARGET DETECTION METHOD}

The basic technology in the intelligent monitoring system is to track and identify the moving vehicles. This technology also occupy a large part of the field of computer vision, causing a large number of experts in related fields to study it, so the technology is developing more and more fast. Since only the monitoring system can mainly identify the vehicles in motion, the vehicle target detection is mainly to solve the problem that the moving objects can be identified and extracted from the static background, and the focus is on the moving targets.

For the quality of the entire monitoring system, the detection of moving targets has played a decisive role. External factors (road conditions of highway, heavy rain, cloudy, etc.) are brought different degree of impact to the vehicle detection, which requires the use of better vehicle target detection methods to try to avoid these external interference. In this way we call it robust to the external environment ${ }^{[1,2]}$.

This section mainly introduces several traditional vehicle target detection methods, including four methods ${ }^{[3]}$ : method based on feature(corner), method based on the difference between the frames, method based on the optical flow field and method based on the background difference. Here are the main ideas of these four methods.

\section{A. Method Based on Feature}

This method is also called corner-based method. Its idea is to find out its special corner from the appearance of things, and then perform the matching operation with the images in the system $^{[4]}$

To complete the matching operation need to go through the following two steps.

- First, extract some representative corners or features from two or more consecutive images detected by the video and create a collection of images for later use.

- Then according to the feature image set obtained in the last step, the structures of the objects corresponding to the corresponding images are matched one by one to complete the calculation of the moving objects to obtain the motion parameters in the coordinate axes. 
After completing these two steps, we can find the corresponding corner for the moving vehicle seen in the video detection, and finish the remaining steps according to the feature in the later target detection.

The feature-based approach sums up well the relevant features of the moving vehicle. However, this method also has limitations, because it is difficult to find the corner of the object and to complete the matching work. In particular, there are many vehicles on the highway, which make it difficult to distinguish between multiple targets.

\section{B. Method Based on the Difference Between the Frames}

The term difference between the frames which is also known as time-domain difference (DT) is a relatively simple method that quickly distinguishes moving objects. The method detects the foreground of the moving target by determining the gray value of the background pixel plus the decisive position.

Generally, the image detected by the video is not threedimensional, but a projection on the two-dimensional plane. The projection changes as the three-dimensional image changes. The magnitude of the difference between consecutive frames of images depends on the transformation of successive scene images in the video. This difference is calculated by extracting the difference between two or more consecutive frames of image.

The point of this method is that the programming complexity is relatively low and the algorithm is easy to implement. It also can solve the influence caused by the external environment interference mentioned in the feature method and has good stability. The disadvantage is that the method is limited by the noise generated by the equipment during the shooting process and also influences the foreground area of the later picture due to the background being blocked so that the formed pixel becomes empty.

\section{Method Based on Optical Flow Field}

The optical flow field is generally due to the shooting movement, which produces an instantaneous velocity field in the movement of the target. In other words, it is the pixel point ${ }^{[5]}$ generated on the surface of the photographed object during its movement. The method based on the optical flow field focuses on the simulation of the object's motion field, first proposed by Gibson ${ }^{[6]}$.This method can check the information of anything under any circumstances, and it is easy to realize the target detection in exercise. However, it cannot be avoided by external force majeure. At the same time, accurate data information cannot be calculated, and a requirement that most systems must meet is not satisfied: real-time.

\section{Method Based on the Background Difference}

The above three methods are typical target detection methods, method based on the background difference is also one of the common methods to achieve target detection ${ }^{[7]}$. The main idea of this method is to construct the background from the detected continuous multi-frame images to obtain the latest background image, and then subtract the background image from the video image to be detected in a certain frame. The larger one is the sport we need to look for.
The method based on background difference is mainly for obtaining efficient background image, so this method depends on the key step of background image extraction. Figure I is the background subtraction extraction prospects of the target out of the process.

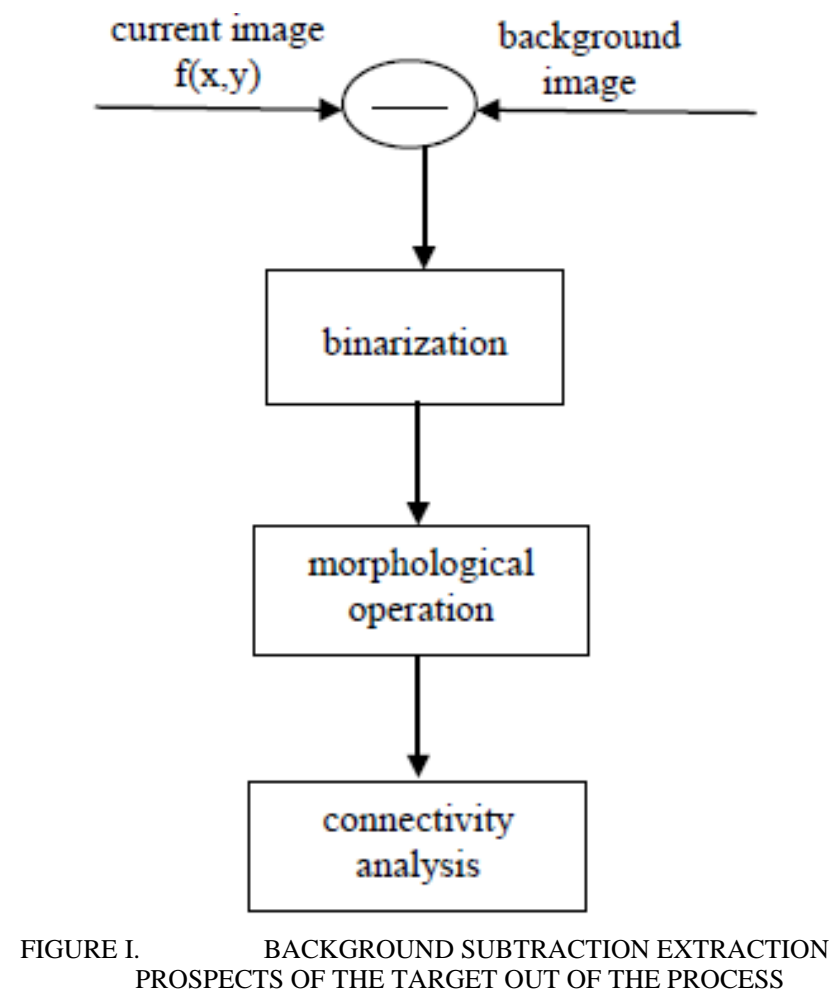

The key to extracting the foreground object by the background subtraction method is how to get the better background image. If the background image is not accurately extracted, then it is impossible to get the accurate foreground object image.

There are basically three methods for background modeling: statistical averaging, filtering, and Gaussian modeling. The statistical averaging method is to perform statistical analysis on the data of the multiple video images and obtain the pixel average as the background pixel value. The more the images are used in this method, the better the effect will be, but at the same time, the higher demand the amount of calculation and the storage amount need. The filtering method is based on the statistical average method to introduce attenuation control factor to update the background image weighted method, this method for the selection of control factors depend greatly. Gaussian background modeling method is the most widely used method for the detection of moving targets and the background modeling algorithm is used in this paper, which is described in detail in the next section.

\section{IMPROVED MiXed GAUSSIAN MODELING MethoD}

Since there are some limitations on the above target detection methods, this section will introduce an improved mixed Gaussian modeling method. Based on the traditional Gaussian modeling, this paper gives an improved method. 


\section{A. Traditional Mixed Gaussian Modeling}

Mono-Gaussian background modeling is based on the background of the pixel formed by the image, and the metric function $\mathrm{f}(\mathrm{x}, \mathrm{y})$ is formed according to the horizontal and vertical coordinates $(x, y)$ where the pixel is located. The value that exceeds the set threshold is used as the background pixel in the model, while the pixels below the threshold will form the foreground pixel.

Mixed Gaussian modeling method is based on the MonoGaussian modeling method based on the completion of at different times, for each pixel to construct a number of measurement functions, such as the measurement function at $t_{1}$ time is $f_{1}(x, y)$, measurement function at $t_{i}$ moment is $f_{i}(x, y)$. The average of all the metric functions at different moments is taken as the Gaussian distribution average and then compared with the threshold.

In all methods, the Gaussian modeling method best meets the background modeling of the highway environment.

\section{B. The Main Drawbacks of Traditional Mixed Gaussian Modeling}

The traditional mixed Gaussian modeling method mainly has the following shortcomings.

- The model requires Gaussian distribution model for each pixel in the image. The calculation of this model will increase as the number of objects used in modeling increases, which leads the hardware to keep up with the system request.

- In general, a vehicle traveling on an highway with a low vehicle density occupies a relatively small proportion of the entire monitoring picture, so that the relatively large occupying area of the vehicle occupies a smaller area. When a frame is Gaussian modeled and updated, the corresponding pixel must be selectively processed.

In view of the above shortcomings, this section will focus on the improved Gaussian modeling method.

\section{Method and Steps of Improved Gaussian Modeling}

In view of the above two points, we make improvements to the traditional mixed Gaussian modeling method, which is to simply optimize the Gaussian background model. Since the optimized model is for vehicles traveling in lane lines and not caring for anything else, we start off with lane lines and accurately lock the vehicles in the lane as long as the lane lines are accurately extracted. The following three steps give the lane line extraction process.

\section{1) Extract lane line boundaries}

Lanes on normal highway are some obvious edges from the direction of image processing. Therefore, in order to get the lane line image, we should first extract the edge of the original image. In addition, the video speed system for the highway shooting direction is either from front to back or from back to front, but not horizontal shooting, so reflected in the image is the lane line in the image is the direction of the vertical direction Therefore, in the edge detection only need to detect the vertical edge. In the related art of image processing, there are many algorithms for edge extraction, but some algorithms are computationally intensive, which is not good for system real-time, such as Canny algorithm. Some algorithms to extract the edge of the effect is not good, have a bad influence on the follow-up processing, such as Laplace algorithm. Considering the efficiency and complexity of the Sobel algorithm ${ }^{[8]}$, it is more suitable for the system.

\section{2) Binarization of Lane Boundary}

In the previous step, we extracted the lane boundary, but the boundary picture of other objects (green belts and fence, etc.) in addition to the lane line will cause interference to us and we must eliminate this interference information to avoid occurrence. There is an error extracting the edge information. According to the pictures taken, we conclude that the gray value of the lane boundary line is higher than the gray value of other objects. Therefore, using the binarization formula, the gray value is calculated. If the gray value is below a given threshold, it is excluded. That is the boundary line of other objects.

\section{3) Extracting the lane boundary line and highway traffic} zone

After step 2), we get the boundary picture we need, but the line in the picture is a part of the original picture. Therefore, we need to use Hough transform algorithm to extract the straight line in front of the lane to complete the repair of the lane boundary line. Although the Hough algorithm has high time complexity and space complexity, this lane edge line only needs to be repaired once, which is negligible for the overall system complexity.

After all the above steps, we can get an edge line that can identify all the vehicle information. In this way, a model is built to track the vehicle's target.

For the practical application of this article, it is only a background of the lane inside the line modeling, so galloping branches on both sides or some other disturbing factors will not have an impact on the background update. Usually, there will be no other interference factors in addition to moving vehicles outside the lane line area, and because the interval time in the continuous between the two video images is too short, except where the vehicle pixel values will have a greater change, the rest of the background area usually does not change much (in the case of sudden changes in the weather), the general idea of updating the Gaussian background model proposed in this paper is as follows.

- According to the two adjacent frames of image difference image, the driving area in the lane line is divided into the moving area (the pixel value is quite different) and the non-moving area.

- For the non-moving region, since the pixel value of the background pixel changes very slowly, it is not necessary to perform a Gaussian model updating operation with a large amount of calculation. Instead, the Gaussian model matching the pixel in the previous frame is directly used for the current frame in. 
- For the motion region, the traditional mixed Gaussian background model must be updated due to the large and irregular changes of pixel values.

According to the improved mixed Gaussian model method, the flow chart of vehicle detection is given as Figure II.

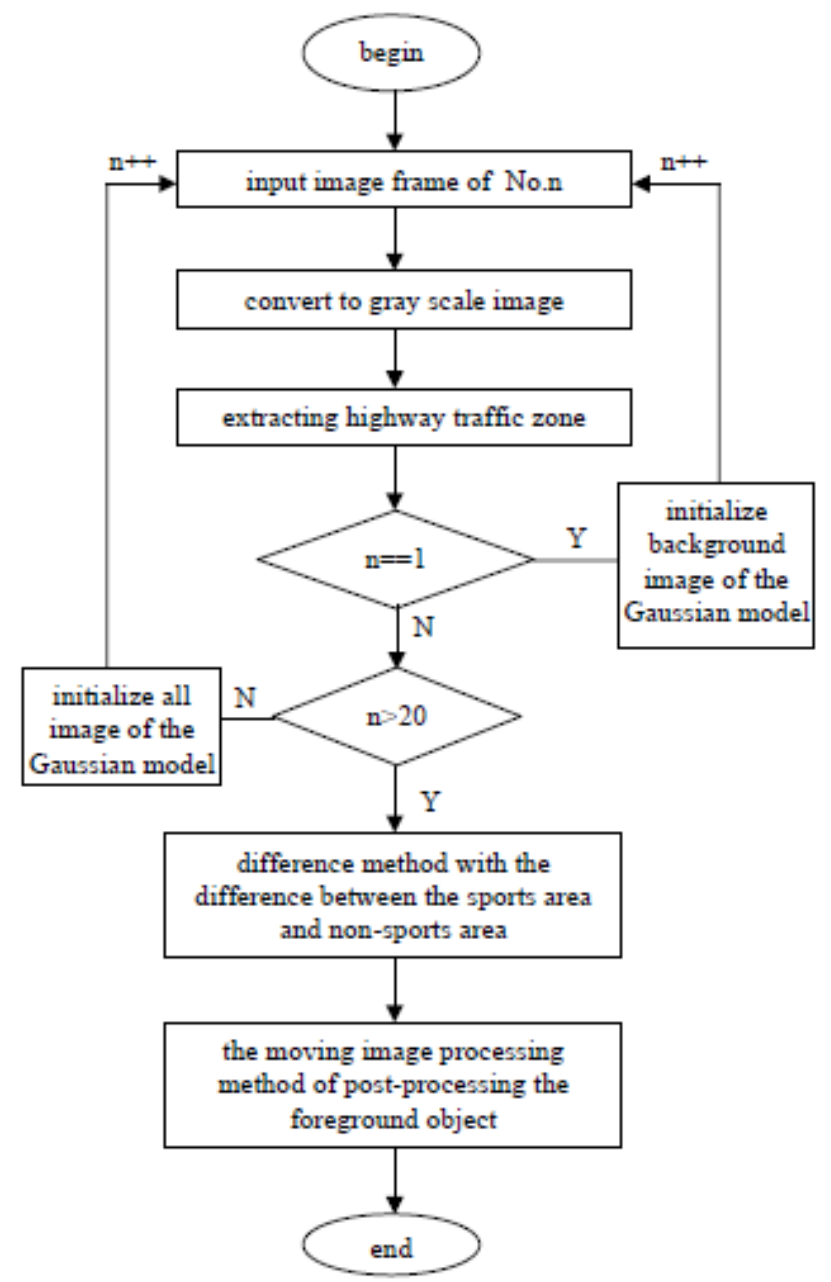

FIGURE II. FLOW CHART OF IMPROVED MIXED GAUSSIAN MODEL METHOD

\section{CONCLUSION}

Intelligent information processing for the highway is now very popular means; it is also a more mature technology, which can provide real-time and accurate access of all of the vehicle information for law enforcement and other relevant departments to give important judgments. The premise of accurate vehicle information acquisition is to track and detect the vehicle targets. In this paper, several popular vehicle target detection methods are introduced and the corresponding advantages and disadvantages are given. Then the method of traditional mixed Gaussian modeling method has been improved correspondingly, and the edge extraction of vehicles in the driving area of highway has been realized in three steps, which provides a solid foundation for vehicle target detection.

\section{ACKNOWLEDGMENT}

The project is supported by Foundation of Jiangxi Provincial Education Department (No.GJJ161309, No.GJJ161304 and No. GJJ151332).

\section{REFERENCES}

[1] Cheng Juan. Motion Target Recognition Algorithm in Complex Background[D], Wuhan, Wuhan University of Technology, 2008.

[2] Paulo A.C. Marques, Jose M.B. Dias, R. Conselheiro Emidio Navarro. Optimal Detection and Imaging of Moving Objects with Unknown Velocity[J]. In Pro. Of the 3rd European Conference on Synthetic Aperture Radar, 2000: 561-564.

[3] Shi Zhiqiang. Motor vehicle speed target detection and tracking technology research and application[D]. Nanchang University, 2007.

[4] Li Guodong, Liu Chunyang, Liu Changan.A Feature-based Dynamic Target Detection and Tracking Algorithm[J] .Journal of Harbin Institute of Technology, 2005,7: 927-929.

[5] Fang Yuqiang, Dai Bin, Song Kanazawa, et al.A method of moving object segmentation based on optical flow and level set[C]. 2009 Automation Conference, 2009,3: 1541-1547.

[6] Chen Wenjie. The Research of the Eliminate Shadow and the Tracking of Multi-objective in Video Speed Measuring System[D]. Nanchang University, 2012.

[7] Gupt S. Modeling O. Martin RFK, et al. Detection and Classification for Vechicles[J], IEEE Transcations on Intelligent Transporatation System. 2002, 3 (1): 37-47.

[8] Tan Xiaojun, Shen Wei, Guo Zhihao. A video-based traffic monitoring method for road traffic[J] .Computer Applications, 2005 (5). 